\title{
Crystallization of supercooled water: A level-set-based modeling of the dendrite tip velocity
}

\author{
A. Criscione ${ }^{\mathrm{a}, *}$, D. Kintea ${ }^{\mathrm{a}}$, Ž. Tuković ${ }^{\mathrm{c}}$, S. Jakirlić ${ }^{\mathrm{a}, \mathrm{b}}$, I.V. Roisman ${ }^{\mathrm{a}, \mathrm{b}, *}$, C. Tropea ${ }^{\mathrm{a}, \mathrm{b}}$ \\ a Institute for Fluid Mechanics and Aerodynamics, Technische Universität, Darmstadt, Germany \\ ${ }^{\mathrm{b}}$ Center of Smart Interfaces, Technische Universität, Darmstadt, Germany \\ ${ }^{\mathrm{c}}$ Faculty of Mechanical Engineering and Naval Architecture, University of Zagreb, Croatia
}

\section{A R T I C L E I N F O}

\section{Article history:}

Received 25 April 2013

Received in revised form 23 July 2013

Accepted 26 July 2013

\section{Keywords}

Supercooled liquid

Level set method

Dendritic crystal growth

Marginal stability hypothesis

\begin{abstract}
A B S T R A C T
'It is well-known that solidification front of a supercooled liquid is unstable; consequently, this instability leads to the appearance of an array of dendrites of sub-micron diameter. The shape and the velocity of the dendrite propagation are determined by the thermodynamic properties of the liquid and solid phases, including interfacial energy as well as the initial temperatures of both. Accordingly, the numerical simulation of solidification process is a rather challenging problem which requires an accurate prediction of high temperature gradients near the moving solidification front. In this study a relevant level set formulation has been developed enabling correct determination of the position and the curvature of the liquid/ solid interface. At this interface a Dirichlet boundary condition for the temperature field is imposed by applying a ghost-face method. For the purpose of updating the level set function and optimizing computing time a narrow-band around the interface is introduced. Within this band, whose width is temporally adjusted to the maximum curvature of the interface, the normal-to-interface velocity is appropriately expanded. The computational model is firstly validated along with the analytical solution of stable freezing. The tip velocity of dendritic patterns (pertinent to unstable freezing) is investigated by performing two-dimensional simulations. The computational results exhibit excellent qualitative and quantitative agreement with the marginal stability theory as well as with the available experiments in the heat-diffusion-dominated region.
\end{abstract}

(c) 2013 Elsevier Ltd. All rights reserved.

\section{Introduction}

Several influencing factors are involved simultaneously in the process of a phase change of pure water: heat transfer, absorption (if a melting process would take place) or release (pertinent to freezing) of latent heat (with possible solute rejection in case of binary substances), surface effects, changes in thermophysical properties with the temperature, etc.

The pure water is supercooled if its temperature becomes lower than the melting point, corresponding to $T_{m}=273.15 \mathrm{~K}$ under atmospheric pressure. This occurs when the energy barrier above which the atoms start to move into the solid lattice structure, required for the formation of a crystal, is not entirely exceeded. Such a state of supercooling is thermodynamically unstable. Correspondingly the liquid is in a metastable state, implying a weak disturbance can initiate a rapid and unstable process of the so-called dendritic solidification. Small perturbations in the initial state can produce

\footnotetext{
* Corresponding authors. Tel.: +49 (0) 6151/16 6553; fax: +49 (0) 6151/16 4754.

E-mail addresses: a.criscione@sla.tu-darmstadt.de (A. Criscione),roisman@sla. tu-darmstadt.de (I.V. Roisman).
}

significant changes in relation to the time-dependent solid/liquid interface. Behavior of such supercooled water drops is rather important for cloud microphysics and understanding of aircraft icing [1,2].

The phenomenon of dendritic crystal growth attracted considerable attention over the last few years. In nature many cases of spontaneous dendritic pattern formation can be found, e.g., in solidification of metals and crystallization of supercooled solutions. The most evident case of dendritic pattern formation are snowflakes which have various types of complex and fascinating shapes. The underlying physics of crystals has been firstly elaborated by Kepler in 1611, [3]. This famous essay about the form of snowflakes represents the first scientific reference to snow crystals. Over three hundred years later, Nakaya [4] has performed a first systematic study about the snow crystals. In this work the crystal morphology created under different environmental conditions has been described. Over the last decades, a number of theoretical and experimental works [5-10], has been published dealing with the detailed macroscopic dynamics of crystal growth (pertaining to non-equilibrium patterns). The common approach to crystallization problems depends on the grade of the supercooling, $\Delta T$ (liquid temperature reduction below its freezing point without solidification), in the 
liquid. At low supercooling $(0.06 \leqslant \Delta T \leqslant 10 \mathrm{~K})$, the freezing process can be considered as a Stefan problem (corresponding to a diffusiondriven growth) [11]. In the case of very low supercooling $(\Delta T<0.06 \mathrm{~K})$, a deviation from the theory can be observed in the experiments. This deviation is assumed to be caused by the effect of natural convection around the crystal promoting its increase in this supercooling range [12]. On the contrary, in the case of increasing supercooling $(\Delta T>10 \mathrm{~K})$, there is a transition from the diffusional to the kinetics-limited growth. Correspondingly, the rate of solidification depends on how fast the liquid molecules can be brought in the proper position, orientation and conformation pertinent to the solid phase.

Recently, Shibkov et al. [13-15] have investigated the free growth of an ice crystal in a supercooled pure water film in a wide initial supercooling range corresponding to $0.1<\Delta T_{0}<30 \mathrm{~K}$. In [14] various shapes of ice crystal patterns in the range between low $\left(\Delta T_{0}=0.1 \mathrm{~K}\right)$ and high $\left(\Delta T_{0}=14.5 \mathrm{~K}\right)$ supercooling have been observed. Evidently, the shape and the velocity of the solidification front depends strongly on the initial supercooling in the pure water film. The shape of dendritic front is determined by the balance between surface energy criterion and the efficiency of the interface (region of negligible thickness where solid and liquid phases coexist) in removing heat. Thus, when pure water freezes in the diffusive regime, different morphologies can appear depending on supercooling and crystalline anisotropy. At higher supercooling, e.g., at $\Delta T_{0}=10 \mathrm{~K}$, transition from diffusional growth to kineticslimited growth is observed. These experimental results have been compared with the theory of dendritic growth of Oldfield and Langer and Müller-Krumbhaar [16-20] exhibiting an excellent agreement within the diffusion-driven growth region. As expected, in the kinetics-limited growth region a certain deviation from the theory is observed.

Mathematically, the phenomenon of solidification can be modeled by utilizing a moving boundary [11]. The heat equation is solved in each phase separately. The temperature fields are coupled through two boundary conditions at the unknown moving boundary between the solid and the liquid phase. The first boundary condition is the velocity of the interface. It depends on how fast the latent heat of solidification is removed from the interface, hence, the velocity can be derived from a heat balance at the interface. The second boundary condition assumes a constant temperature at the interface corresponding to the melting temperature. Locally, the melting temperature at the interface will be altered by an amount depending on the surface tension between the solid and liquid phases and the local curvature, in line with the GibbsThomson effect. Another effect to be taken into consideration are the density changes due to temperature variation. These changes induce flow resembling the natural convection in the presence of gravity which affects the heat transfer in the liquid.

The direct solution of the time-dependent Stefan problem represents a great challenge. An appropriate front tracking method accounting for the moving solid-liquid interface is required. Over the last decades, the phase-field models have been successfully applied for the simulation of dendritic growth. The basic idea behind phase-field methods is to artificially thicken the interface to an extent which can be resolved by the numerical mesh. This thickening procedure is accomplished by introducing the so-called "order parameter" for each cell, ranging between zero and unity. If the order parameter takes the value of unity the corresponding grid cell is filled entirely with the liquid phase. In contrast, the zero value of the order parameter indicates the grid cell comprising completely the solid phase. The position of the interface coincides with the surface whose order parameter takes the value 0.5 . The width of the artificially thickened interface corresponds to the area within which the order parameter varies smoothly from zero to unity. The outcome is a smeared interface over which all governing equations can be solved taking into account appropriately averaged (i.e. weighted) material properties. This allows application of the physical models within such a diffuse interface. By doing so, the computational difficulties pertinent to the tracking of a sharp interface are avoided to a great extent. This contributes to the high popularity of the phase-field methods for simulating dendritic solidification [21-25]. Although the phase-field modelling approach has been shown to be very useful in investigating solidification patterns, there were still some important drawbacks which should be addressed. The principal drawback is the non-physical representation of the diffuse interface. The width of the interface represents an adjustable parameter, which may also lead to unphysical interactions. Unless a field equation solver is developed to enforce the conservation of energy for a control volume positioned strictly at the interface, the interfacial velocity will be inaccurately obtained.

The presently adopted level set method is a computational approach aiming at overcoming the limitations of phase-field models with respect to the interface surface tracking; here the boundary movement is tracked implicitly. This method, firstly introduced by Osher and Sethian [26], describes the spatial distribution of a level set function, $\Phi$, within the entire solution domain. The solid-liquid interface is represented by the zero-value contour of the level set function, which is governed by its own advection-type equation of motion. These equations providing a sharp interface are solved directly and can be handled in a straightforward manner. Although the level set methods still did not reach the popularity the phasefield methods have for studying crystal growth phenomena, they have been increasingly applied to several problems involving moving boundaries [27-29] and crystallization [23,30,31] exhibiting good predictive performance in returning qualitative features of the dendrites.

In this work, an extended level set method using a ghost-face algorithm for solving the temperature field is presented. Unlike the conventional level set method, this extended version converges to exact solution of the Stefan problem for planar solidification. Concerning the tip velocity of dendritic growth within the diffusion-driven growth region the results of two-dimensional simulations show excellent qualitative and quantitative agreement with the experiments of Shibkov [13-15] and the theory of Oldfield [16] and Langer and Müller-Krumbhaar[17-20]. Hence, the computational model is capable of capturing both stages of crystallization: the first rapid, dendritic-like growth (corresponding to the phenomenon of unstable freezing) and the second planar-shaped growth stage (associated with the stable freezing process).

\section{Theoretical background}

The common approach to a freezing problem consists in its consideration as a two-phase Stefan problem. The term "two-phase" refers here to the phases taking an "active" part in the process. Accordingly, both the liquid and the solid phases are active, i.e. the heat conservation is solved in both sub-domains. Let us consider a square domain, $D$, of pure water where at every time step and at every numerical node the water is either in the liquid (supercooled) state or in the solid state. Let $T(\boldsymbol{x}, t)$ represents the temperature of the water. The region where the water appears as solid is denoted by $\boldsymbol{\Omega}_{s}$ and the region where the water is a liquid by $\boldsymbol{\Omega}_{l}$. The interface between the solid phase and the liquid phase is of infinitesimal thickness and is denoted by $\boldsymbol{\Xi}$.

\subsection{Governing equations}

As the flow in liquid region is not considered presently, the energy equation describing time dependent heat conduction in both regions reduces to: 
$\rho_{s} c_{v, s} \frac{\partial T}{\partial t}=\nabla \cdot\left(k_{s} \nabla T\right), \quad \boldsymbol{x} \in \boldsymbol{\Omega}_{s}$,

$\rho_{l} c_{v, l} \frac{\partial T}{\partial t}=\nabla \cdot\left(k_{l} \nabla T\right), \quad \boldsymbol{x} \in \mathbf{\Omega}_{l}$,

with the indices $s, l$ denoting the solid and the liquid phase, respectively. At the interface $\boldsymbol{\Xi}$ between the liquid and the solid phase the energy balance leads to the Stefan condition

$\rho L v_{n}=\left(k_{l} \nabla T_{l}-k_{s} \nabla T_{s}\right) \cdot \boldsymbol{n}, \quad \boldsymbol{x} \in \mathbf{\Xi}$,

where $L$ is the latent heat released by the phase change, $\boldsymbol{n}$ is the unit normal vector directed towards the liquid region and $v_{n}$ denotes the solidification speed. Eq. (3) expresses dependency of the local normal velocity of the interface on the heat flux discontinuity at the interface. In the case of a curved interface, the temperature at the interface, $T_{f}$, needs to be described by utilizing the Gibbs-Thomson relation, which in the case of $c_{s}=c_{l}$ can be written in the form

$T_{f}=T_{m}-\Gamma \kappa, \quad \Gamma=\frac{\sigma T_{m}}{\rho L}, \quad \boldsymbol{x} \in \boldsymbol{\Xi}$

with $\sigma$ representing the interfacial energy, $\kappa$ the curvature of the interface and $\Gamma$ denoting the capillary constant. The densities of the liquid and the solid phases in our case are assumed equal, $\rho:=\rho_{l}=\rho_{s}$. The kinetic effects in Eq. (4) are neglected in the present study. It should be recalled, that for the solid interface curvatures whose radii are larger than several micrometers, the Gibbs-Thomson effect becomes also negligibly small and the interface temperature approaches the melting temperature, $T(\boldsymbol{x}, t) \approx T_{m}$.

For materials with different heat capacities and densities the interface temperature in Eq. (4) can be rewritten as follows [32]

$T_{f}=T_{m}\left[1-\bar{\Gamma}\left(1+T_{m}\left(c_{s}-c_{l}\right) \bar{\Gamma}\right)\right]$

with

$\bar{\Gamma}=\frac{\sigma \kappa}{\rho_{s} L}-\left(\frac{1}{\rho_{l}}-\frac{1}{\rho_{s}}\right) \frac{p_{l}-p_{0}}{L}$,

where $p_{l}$ represents the pressure in the liquid phase, whereas $p_{0}$ denotes the ambient pressure.

\subsection{Stable freezing: propagation of a flat interface}

For the purpose of derivation of the analytic solution of the present problem we first consider the one-dimensional case of stable freezing [11]: a semi-infinite slab occupied by the supercooled liquid at $T_{l}^{0}<T_{m}$, with $T_{l}^{0}$ representing the initial temperature of the liquid. At initial time, $t=0$, the condition $T_{s}^{0}<T_{m}$ is imposed on the slab at $x=0$. Assuming constant densities, $\rho_{s}=\rho_{l}:=\rho$ and freezing at the equilibrium melting temperature, $T_{m}$, the solution for the time-dependent thickness of the solid front $X(t)$ reads

$X(t)=2 \lambda \sqrt{\alpha_{s} t}$,

where $\lambda$ is dependent on the Stefan number and $\alpha_{s}$ is the thermal diffusivity in the solid phase. The solution for the temperature in the solid and liquid phase, respectively, is defined as follows

$T(\boldsymbol{x}, t)=T_{s}^{0}+\left(T_{m}-T_{s}^{0}\right) \frac{\operatorname{erfc}\left(\frac{\boldsymbol{x}}{2 \sqrt{\alpha_{s}}}\right)}{\operatorname{erf}(v \lambda)}, \quad \boldsymbol{x} \in \Omega_{s}$,

$T(\boldsymbol{x}, t)=T_{l}^{0}+\left(T_{m}-T_{l}^{0}\right) \frac{\operatorname{erf}\left(\frac{\boldsymbol{x}}{2 \sqrt{\alpha_{l} t}}\right)}{\operatorname{erf}(\lambda)}, \quad \boldsymbol{x} \in \Omega_{l}$,

where $v=\sqrt{\alpha_{s} / \alpha_{l}}$.

\subsection{Mullins-Sekerka morphological stability analysis}

During the solidification process of the supercooled liquid the interface between the solid and the liquid phase becomes unstable due to the inherent destabilizing effect of supercooling. On the other hand, the forces associated with the surface tension at the ice interface act towards the interface stabilization. The balance between these two effects can be analyzed by the classical approach to morphological stability introduced by Mullins and Seker$\mathrm{ka}$ in the context of directional solidification $[8,9]$. By utilizing this approach one can study the stability of a flat-interface solution under small perturbations. The derivative of the amplitude of the sinusoidal perturbation with respect to time reads

$\delta^{\prime}(t)=-\Lambda(\omega) \delta(t)$

with

$$
\Lambda(\omega)=\omega[\underbrace{\Gamma \omega^{2}\left(k_{l}+k_{s}+v_{0} \rho \Delta c\right)}_{\text {Gibbs-Thomson }}+\underbrace{k_{l}(\nabla T)_{l}+k_{s}(\nabla T)_{s}}_{\text {Supercooling }}] / \rho L .
$$

Here $v_{0}$ is the magnitude of the constant interface velocity of the unperturbed flat interface. For a positive $\Lambda$, the perturbations will decay exponentially, whereas for a negative value of $\Lambda$ the pertubations will grow exponentially. The perturbations will remain theretically at their initial value if $\Lambda=0$.

The first term on the right-hand side of Eq. (11), $\Gamma \omega^{2}\left(k_{l}+k_{s}+\right.$ $v_{0} \rho \Delta c$ ), describes the stabilizing influence of the interfacial energy. It is unconditionally positive and grows quadratically with the frequency of the perturbations. The sum of the two heat fluxes, $k_{l}(-$ $\nabla T)_{l}+k_{s}(\nabla T)_{s}$, can become negative, if the liquid is supercooled. Thus, it has a destabilizing influence on the solidification front. If the surface tension takes the value zero, the interface is unconditionally unstable for supercooled solidification. Eq. (11) represents the basis for the computation of the cutoff wavelength $\lambda_{c}$, which is the largest possible wavelength for a stable interface. It is derived by setting $k_{s}=k_{l}=k, c_{s}=c_{l}=c, \rho_{s}=\rho_{l}=\rho$ and equating the whole expression with zero, since $\Lambda=0$ matches the stability limit. This finally yields the cutoff wavelength

$\lambda_{C}=2 \pi \sqrt{\frac{2 \alpha}{v_{n}} \cdot \frac{T_{m} \sigma c_{v}}{L_{v}^{2}}}=2 \pi \sqrt{\delta_{D} \delta_{C}}$.

Here, $\delta_{D}=2 \alpha / v_{n}$ represents the diffusion length and $\delta_{C}=T_{m} \sigma c_{v} / L_{v}^{2}$ the capillary length, with index $v$ referring to the volume of the material.

\subsection{Unstable freezing: dendritic growth}

If the crystallization front becomes morphologically unstable it means that the destabilizing effect in Eq. (11) starts to dominate the system, the small perturbations at the interface will evolve into the formation of various polycrystal structures, in particular, dendritic ones (depending on the supercooling range). Analytical treatments approached the problem of dendritic growth by assuming a mathematically definable shape such as a parabola of revolution or an ellipsoid, resulting in a solution of the diffusion problem subjected to the complicated boundary conditions at the crystal interface. Ivantsov [5] derived an analytical solution for the steady state tip velocity and tip radius of paraboloidal-type crystals under the assumption that, firstly, the kinetics is instantaneous, and secondly, the interfacial energy takes a zero $(\sigma=0)$ value. Accordingly, this theory considers a single needle with a parabolic tip shape growing into an infinite half-space of a supercooled liquid. Indeed, Ivantsov adopted a mathematically definable shape of the growing crystal tip which is demonstrated to be close to a realistic constellation [13-15]. The Péclet number, $P e$, derived from the Ivantsov's 
theory is used to establish a correlation between the tip velocity, $v_{t}$, and the tip radius, $r_{t}$ :

$P e=\frac{r_{t} v_{t}}{2 \alpha}$.

There is a unique Péclet number for every dimensionless supercooling, $S t^{-1}=c_{l} \Delta T_{0} / L$, with $\Delta T_{0}$ representing the initial supercooling. This Péclet number is obtained by setting the Ivantsov function to $S t^{-1}$

$I(P e)=P e \cdot e^{P e} E_{1}(P e)=S t^{-1}$,

where $E_{1}$ is the exponential integral,

$E_{1}(\xi)=\int_{\xi}^{\infty} z^{-1} e^{-z} d z$

Eq. (14) correlates the Péclet number, Pe, with the dimensionless supercooling, $S t^{-1}$. The solution of the Ivantsov function describes a family of parabolas/paraboloids for a given $\Delta T$. In Fig. 1(a) the Ivantsov solution for a supercooling of $\Delta T=10 \mathrm{~K}$ is displayed as example. The Ivantsov prediction for $r_{t} v_{t}$ is well verified experimentally; Huang and Glicksman [33,34] have shown that for a given $\Delta T$, all observed needles lie on the Ivantsov curve. Oldfield [16] and Langer and Müller-Krumbhaar [17] suggested that for a given steady needle with appropriate surface energy, a condition of stability should be invoked. If accounting only for the surface energy, $\sigma$, by neglecting the undercooling, a new dimensionless parameter

$\epsilon=\frac{\delta_{C} \delta_{D}}{r_{t}^{2}}=\left(\frac{\lambda_{C}}{2 \pi r_{t}}\right)^{2}$

is defined, representing the so-called controlling parameter for the "operating point" of the needle. Assuming that the emerging needle tip radius, $r_{t}$, corresponds exactly to the cutoff wavelength, $\lambda_{C}$, coping with stability of a planar interface via the Mullins-Sekerka criterion Eqs. (12) and (16) can be evaluated as

$\epsilon^{*}=\frac{1}{4 \pi^{2}} \approx 0.025$.

(a)

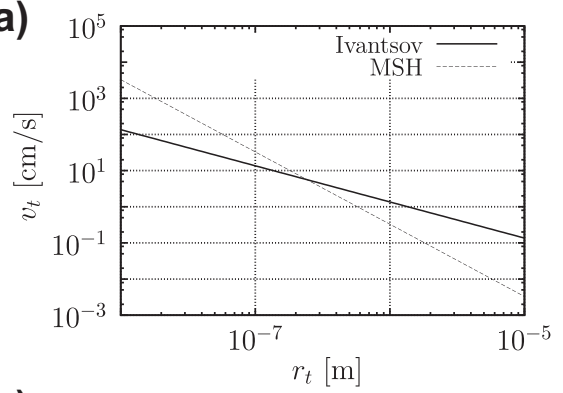

(b)

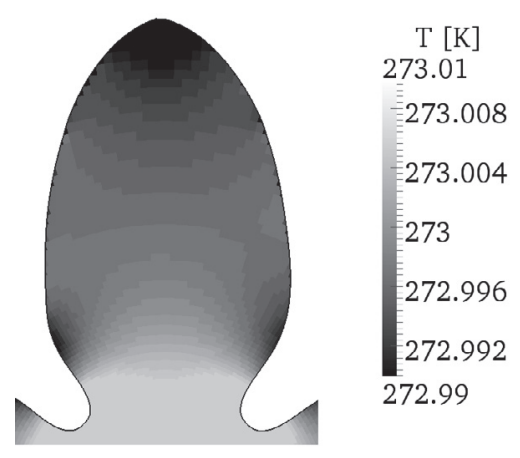

Fig. 1. (a) Ivantsov solution and curve for the marginal stability hypothesis (MSH) and (b) temperature distribution of an isolated crystal growing into supercooled water.
The computations reported by Langer and Müller-Krumbhaar [19] show that

$\epsilon^{*}=0.020 \pm 0.007, \quad($ for $2 \mathrm{D})$,

$\epsilon^{*}=0.025 \pm 0.007, \quad($ for $3 \mathrm{D})$.

If the controlling parameter, $\epsilon$, deviates from the operating point, $\epsilon^{*}$, either the side-branching instability or the tip instability drives $\epsilon$ towards $\epsilon^{*}$. Thus, the operating point is at $\epsilon^{*}$ or close to $\epsilon^{*}$, yields finally, together with Eq. (16), a correlation between tip velocity, $v_{t}$, and its radius, $r_{t}$.

In the case of solidifying water with an initial supercooling of $\Delta T=10 \mathrm{~K}$ (Fig. 1(a)), the emerging crystal is determined by the intersection point of the Ivantsov curve and the marginal stability curve. In this case the dendrite grows at a velocity of $5.61 \mathrm{~cm} / \mathrm{s}$ to a tip radius of $r_{t}=2.42 \cdot 10^{-7} \mathrm{~m}$. The shape of the needle is not paraboloidal anymore if surface energy is accounted for, but the deviation from such a shape is assumed to be small $[19,20]$.

\section{Computational algorithm}

The computational algorithm has been developed which allows to solve numerically the energy Eqs. (1) and (2), which satisfy the matching conditions at the moving interface, determined by Eqs. (3) and (4). The position of the liquid/solid interface is accurately determined using the level-set approach. This ensures the high possible precision in the estimation of the temperature gradients near the interface and the interface curvature, required for the calculation of the velocity propagation of the solidification front in Eqs. (3) and (4).

\subsection{Level set approach}

The basic idea behind the level set method is to use an iso-contour of a particular function defining the surface; accordingly, the interface is located where this function amounts a certain value [26]. The level set equation

$\frac{\partial \Phi}{\partial t}+\boldsymbol{W} \cdot \nabla \Phi=0$

is used to track the interface location constituting the set of points identified by $\Phi=0$. The liquid and solid phases are denoted by the points fulfilling the criteria $\Phi>0$ and $\Phi<0$, respectively. It is convenient to define the level set function as a signed distance function. The so-called zero level set function describes the interface, whereas the value of the outer level set field represents the distance to the interface. The sign of the latter indicates the side of the interface one looks at. This simplifies the expressions for the normal-tothe-interface gradients as well as the surface curvature.

\subsection{Finite volume discretization of the thermal energy equation}

The heat transfer equation in the solid phase, Eq. (1), is solved in the sub-domain indicated by $\Phi<0$ independently of the solution procedure for the liquid phase. Subsequently, Eq. (2) is solved in the sub-domain identified by $\Phi>0$. The same is valid in opposite direction. Hence, two different temperature fields are calculated for both the liquid phase and the solid phase. Ghost-faces are introduced which separate the cells in the liquid sub-domain from the cells in solid phase, Fig. 2(left). Accordingly, to each individual ghost-face in the domain a corresponding ghost-point is to be assigned, Fig. 2(right), whose position vector is defined by the intersection of the zero level set function and the connection vector of the cell-centers, $P_{i, j}$ and $P_{i+1, j}$. The temperature at the interface surface, $T_{f}$, is imposed as a Dirichlet boundary condition for both temperature fields, i.e. at both sides of interfaces, in the liquid and in 

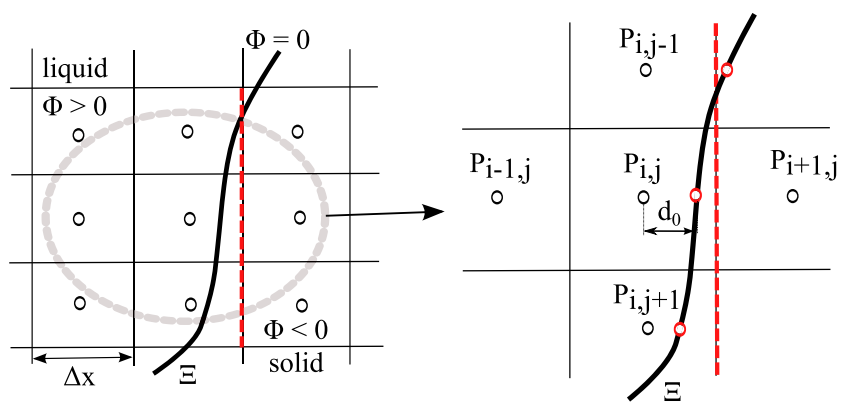

$\circ$ ghost point

--- ghost face

Fig. 2. Finite volume discretization in a two-dimensional domain.

the solid phase. Thus, the temperature at the ghost-points is set as $T_{g p}=T_{f}$. The temporal and spatial discretization of the energy equation in the liquid phase, using Euler and the central differencing scheme, leads to

$$
\begin{aligned}
\rho_{l} c_{v, l} \frac{T_{i, j}-T_{i, j}^{0}}{\Delta t} V_{i, j}= & k_{l} \frac{T_{g p}-T_{i, j}}{d_{0}} S_{i, j}^{i+1, j}+k_{l} \frac{T_{i-1, j}-T_{i, j}}{d_{i, j}^{i-1, j}} S_{i, j}^{i-1, j} \\
& +k_{l} \frac{T_{i, j+1}-T_{i, j}}{d_{i, j}^{i, j+1}} S_{i, j}^{i, j+1}+k_{l} \frac{T_{i, j-1}-T_{i, j}}{d_{i, j}^{i, j-1}} S_{i, j}^{i, j-1},
\end{aligned}
$$

where $T_{i, j}^{0}$ is the value stored at the grid node $P_{i, j}$ at the previous time step and $V$ represents the cell volume, $S$ is the face area. The index gp denotes the ghost-points.

To achieve the required format of the equation system

$[A] \boldsymbol{T}=\boldsymbol{C}$,

the diffusive ghost-face term in Eq. (21) is decomposed as follows

$\left(\frac{\rho_{l} c_{v, l} V_{i, j}}{\Delta t}+\frac{k_{l} S_{i, j}^{i+1, j}}{d_{0}}+\frac{k_{l} S_{i, j}^{i-1, j}}{d_{i, j}^{i-1, j}}+\frac{k_{l} S_{i, j}^{i, j+1}}{d_{i, j}^{i, j+1}}+\frac{k_{l} S_{i, j}^{i, j-1}}{d_{i, j}^{i, j-1}}\right) T_{i, j}$

$+\frac{k_{l} S_{i, j}^{i-1, j}}{d_{i, j}^{i-1, j}} T_{i-1, j}+\frac{k_{l} S_{i, j}^{i, j-1}}{d_{i, j}^{i, j-1}} T_{i, j-1}+\frac{k_{l} S_{i, j}^{i, j+1}}{d_{i, j}^{i, j+1}} T_{i, j+1}=\rho_{l} c_{v, l} T_{i, j}^{0} \Delta t V_{i, j}+\frac{k_{l} T_{g p}}{d_{0}} S_{i, j}^{i+1, j}$

The assumption $T_{f}=T_{m}$ is valid only for planar and infinitely slow interfaces. In order to account for the curvature dependency on the interface temperature, Eq. (4), it is necessary to calculate the curvature itself. Under the assumption that the curvature represents a constant within the computational cell with the center node $P_{i, j}$, the curvature radius of the zero level set contour (interface) at the cell-center point is computed as $r_{i, j}=1 / \kappa_{i, j}$, where the curvature $\kappa$ is computed as follows

$\kappa=\nabla \cdot \frac{\nabla \Phi}{|\nabla \Phi|}$.

Subtracting the normal distance between the cell center and the interface, $\Phi_{i, j}$, from the curvature radius, $r_{i, j}$, one obtains:

$r_{f}=r_{i, j}-\Phi_{i, j}$

as shown in Fig. 3(a). The interface curvature radius, $r_{f}$, is used to compute the curvature at the interface, $\kappa_{f}=1 / r_{f}$. The interface temperature, $T_{f}$, is calculated according to the Gibbs-Thomson relation, Eq. (5).

Accurate values of the normal derivative, $\nabla T \cdot \boldsymbol{n}$, are necessary at grid nodes close to the interface. For this reason ghost-faces and corresponding ghost-points are used. Appointing the value of the temperature at all ghost-points one may perform, for the scenario depicted in Fig. 2 (right), a linear extrapolation (if considered from the liquid phase) or interpolation (if observed from the solid phase) for calculating the temperature at the ghost-face. From the
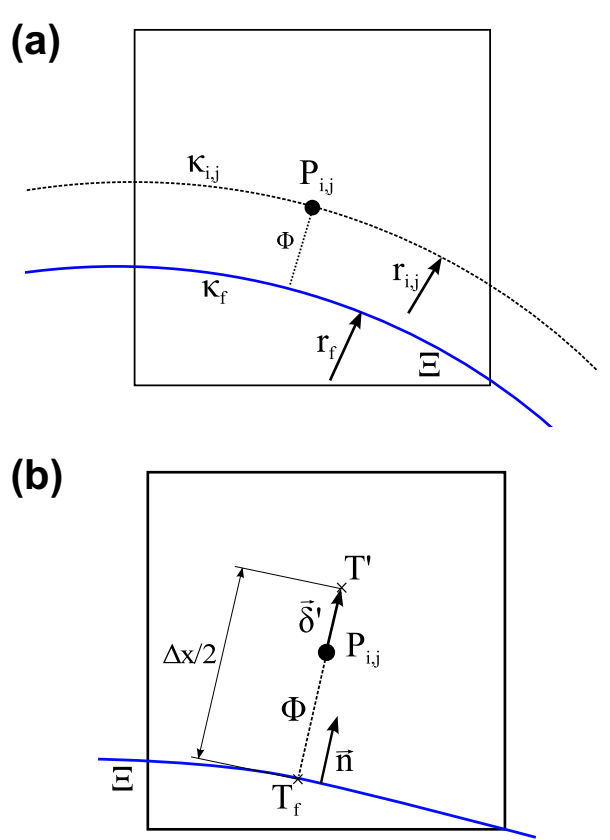

Fig. 3. Schematic illustrating the computation (a) of the curvature and (b) of the temperature normal derivative at the interface.

solid sub-domain, the temperature at the ghost-face, $T_{g f}^{s}$, is determined by linear interpolation between the temperature value stored at $P_{i+1, j}$ and the ghost-point. Whereas $T_{g f}^{l}$ is calculated by linear extrapolation from $P_{i, j}$ and the corresponding ghost-point. Afterwards one may calculate the normal derivative of the temperature close to the interface from both the liquid side and the solid side using the temperature stored at the cell-centers and the calculated values stored temporarily at the ghost-faces. The temperature $T^{\prime}$ is calculated in a virtual point within the current cell (Fig. 3(b)), which is distant half cell length, $\Delta x / 2$, normal to the interface

$T^{\prime}=T_{i, j}+\boldsymbol{\delta}^{\prime} \cdot(\nabla T)_{i, j}$

Here, $(\nabla T)_{i, j}$ represents the temperature gradient at the cell-center node $P_{i, j}$. $(\nabla T)_{i, j}$ needs the values stored at the ghost-faces to be computed. The vector $\boldsymbol{\delta}^{\prime}$ is defined as

$\boldsymbol{\delta}^{\prime}=\boldsymbol{n}\left[\frac{\Delta x}{2}-\Phi_{i, j}\right]$

which ensures, that the distance from the interface to the point where $T=T^{\prime}$ corresponds exactly to $\Delta x / 2$. Therefore, the interfacenormal derivative is approximated as follows

$\nabla T \cdot \boldsymbol{n} \approx \frac{T^{\prime}-T_{f}}{\Delta x / 2} \cdot \boldsymbol{n}$

In order to avoid numerical instabilities we expand this procedure utilizing the following restriction: if the distance from the grid node $P_{i, j}$ or $P_{i+1, j}$ to the interface, $\delta$, is smaller than $\Delta x^{2}, \delta$ will be assumed to be $\Delta x^{2}$ [35]. After having calculated $\nabla T \cdot \boldsymbol{n}$ at all grid nodes next to the interface the value of the normal derivative at the grid node $P_{i, j}$ is averaged over the corresponding phase side within an area, whose width accommodates three interface cells in the closest neighborhood of the cell under investigation. In this way, griddependent numerical errors are reduced. 
3.3. Extension of the interface velocity and update of the level set function

The normal-to-interface velocity, $\boldsymbol{v}_{\boldsymbol{n}}$, may be estimated in accordance with the fulfillment of the Stefan condition, Eq. (3). Hence, the interface position changes dynamically after each time step; it is evolved in time from $\Phi^{n}$ to $\Phi^{n+1}$ using $\boldsymbol{v}$ and a first order Euler time-stepping method.

This makes it necessary to alter the level set function after each time step in order to update it to a signed distance function. This update is done by extending the interface velocity in the normalto-interface direction within a narrow-band around the interface. The width of the narrow-band, $w_{n b}$, is calculated at each time step as

$w_{n b}=\beta \frac{1}{\kappa_{\max }^{*}}$,

where $\kappa_{\max }^{*}$ is the maximum curvature of the interface and $\beta$ represents a system dependent parameter of order $O(1)$. The narrowband increases the efficiency and stability of the algorithm. The interface velocity is expanded in the direction normal to the interface, $\boldsymbol{N}=\Phi /|\Phi|$, within the band accommodating both the liquid and the solid side according to

$\boldsymbol{v}_{\text {ext }, t}+\boldsymbol{N} \cdot\left(\nabla \boldsymbol{v}_{\text {ext }}\right)=0$.

The numerical time discretization given by

$\frac{\boldsymbol{v}_{e x t}^{n}-\boldsymbol{v}_{e x t}^{n-1}}{\Delta t}+\boldsymbol{N} \cdot\left(\nabla \boldsymbol{v}_{e x t}\right)^{n-1}=0$,

yields

$\boldsymbol{v}_{e x t}^{n}=\boldsymbol{v}_{e x t}^{n-1}+\boldsymbol{N} \cdot \nabla \boldsymbol{v}_{e x t}^{n-1} \Delta t$.

Here, $\nabla \boldsymbol{v}_{\text {ext }}$ is discretized locally with a propagating direction defined by $\gamma \boldsymbol{N}$, where $\gamma=1$ for the liquid side and $\gamma=-1$ for the solid side. The time step, $\Delta t$, is adaptively corrected using the CourantFriedrichs-Lewy (CFL) condition, with the CFL number being set to Co $\leqslant 0.5$.

To retain the values of $\Phi$ close to those of a signed distance function, $|\nabla \Phi|=1$, within the band, the level set function is updated according to

$\Phi^{n}=\Phi^{n-1}-\left(\boldsymbol{v}_{e x t}^{n} \cdot(\nabla \Phi)^{n-1}\right) \Delta t$,

with $\Delta t$ being also adaptively corrected ( $C o \leqslant 0.5$ ).

After the update of the level set function within the narrowband, the outer level set function has to be reinitialized as follows

$\Phi_{\tau}+S\left(\Phi_{0}\right)(|\nabla \Phi|-1)=0$

to enable the setting a new band around the interface in the next time step. Eq. (34) is iterated for a few steps within a fictitious time, $\tau . S\left(\Phi_{0}\right)$ is a smoothed-out sign function.

\section{Results}

\subsection{Stable freezing: planar solidification front}

Eqs. (2) and (3) are solved firstly aiming at verification of their implementation into the computational code OpenFOAM ${ }^{\varpi}$ for a supercooled one-dimensional solidification, keeping in mind the possibility to compare the results with a corresponding analytical solution. Let $T_{s}^{0}=T_{l}^{0}=250 \mathrm{~K}$ and $\alpha_{s}=\alpha_{l}=1.427 \cdot 10^{-7} \mathrm{~m}^{2} / \mathrm{s}$ (thermal diffusivity of water). The boundary at $x=0$ is held at a constant temperature of $250 \mathrm{~K}$. Fig. 4(a) shows the position of the interface for different grid resolutions. With a decreasing cell size the accuracy of the approximation increases linearly. In Fig. 4(b) the numerical approximation for the temperature distribution is

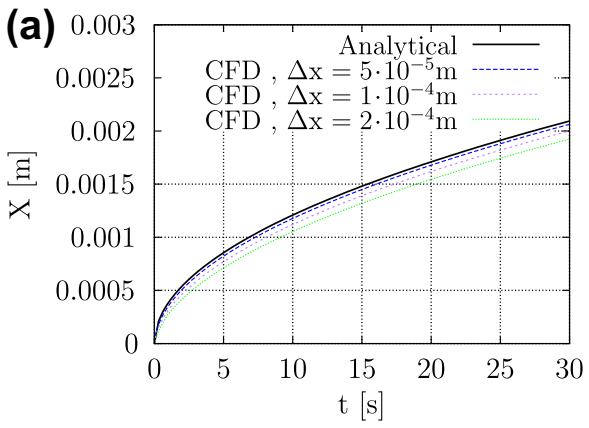

(b)

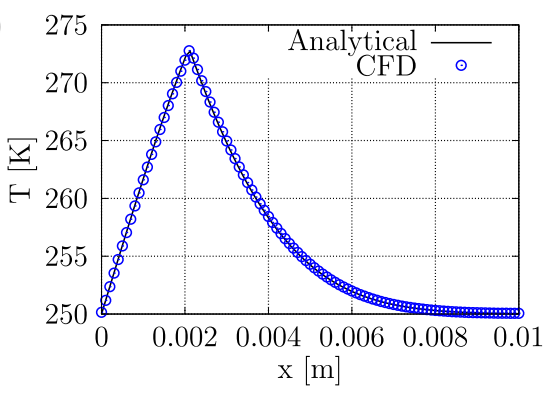

Fig. 4. Planar solidification front: (a) position of the interface and (b) temperature distribution at $t=30 \mathrm{~s}$

depicted by way of example at time instant $t=30 \mathrm{~s}$ and compared to the analytic solution. The numerical solution has been obtained with a cell size of $\Delta x=5 \cdot 10^{-5} \mathrm{~m}$. Accordingly, the implemented algorithm is capable of returning accurately the analytic solution for stable freezing.

\subsection{Curved solidification front}

For a curved interface we have to validate our numerical code along with the Mullins-Sekerka morphological stability analysis. We introduce a small sinusoidal perturbation. If imposing no supercooling or only a low supercooling to the fluid, the interface stays stable, i. e. the amplitude of the perturbation decays exponentially, Fig. 5(a), whereas the amplitude rises if the supercooling reaches a critical value, Fig. 5(b). The figure illustrates comparison between numerically and analytically obtained amplitude of the perturbation over time. Fig. 5(b) reveals the exponential growth of the perturbations in case the supercooling is higher than the critical value, corresponding to a non-stable state of the flat interface. The increasing departure of the numerical solution from the analytical one as time progresses, Fig. 5(b), is due to the fact that one assumption behind the analytical solution implies the interface remaining unperturbed. A slight but progressive departure of the computationally obtained interface position from the analytically determined one, corresponding closely to the situation when the perturbation amplitudes are growing, is in accordance with increasing error of the analytical solution.

If the frequency of the perturbation is increased in the case of an unstable interface it will, to a certain extent, make the interface stable again, representing the outcome of the interfacial energy (by relevance to the Gibbs-Thomson relation). This effect is accounted for in the present numerical algorithm. Fig. 1(b) displays the temperature distribution at the solidification front of an ice crystal growing into supercooled water. Accordingly, the mesh resolution is of the same order of the capillary length scale, $\Delta T_{v}=\sigma \kappa /$ $\left(L \rho_{l}\right)$, which enables correct representation of the physical reality. The temperature at the convex front portion is lower than the melting temperature, $T_{m}$. This temperature reduction has a 

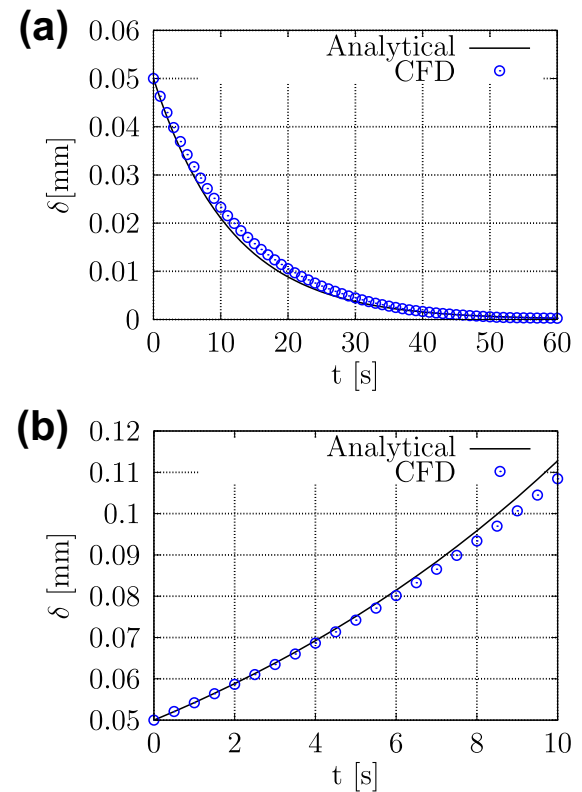

Fig. 5. Curved solidification front under initial small perturbations $\mathrm{Y}=\delta_{0} \sin \omega x$ with $\delta_{0}=5 \cdot 10^{-5} \mathrm{~m}$ and $\omega=6.3 \cdot 10^{3} 1 / \mathrm{s}$. (a) Decaying magnitude of initial perturbation for not supercooled water: $(\nabla T)_{l}=3685 \mathrm{~K} / \mathrm{m},(\nabla T)_{s}=1000 \mathrm{~K} / \mathrm{m}$. (b) Exponential growth of the initial perturbation for large supercooling: $(\nabla T)_{l}=$ $-3685 \mathrm{~K} / \mathrm{m},(\nabla T)_{s}=-1000 \mathrm{~K} / \mathrm{m},-(\nabla T)_{l}<k_{s} / k_{l} \cdot(\nabla T)_{s}$.

stabilizing effect on the interface. Contrary to that, the temperature at the interface is increased in the case of the concave front shape.

\subsection{Unstable freezing: tip velocity of growing dendrites}

The Mullins-Sekerka morphological stability analysis, Eq. (11) describes, under which circumstances perturbations of the interface decay, Fig. 5(a), grow, Fig. 5(b), or remain at their initial value. If the perturbations grow, dendrites or needle crystals will emerge. Neglecting the interfacial energy, the product of the tip velocity $v_{t}$

(a)

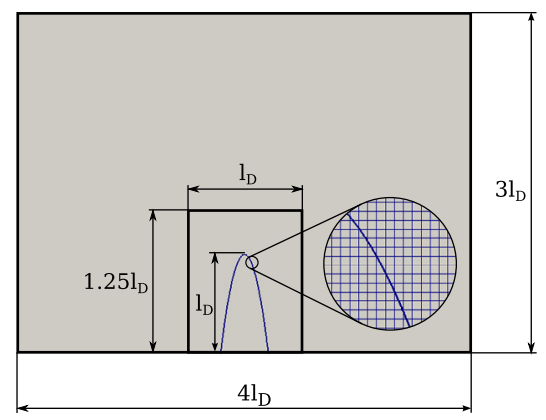

(b)

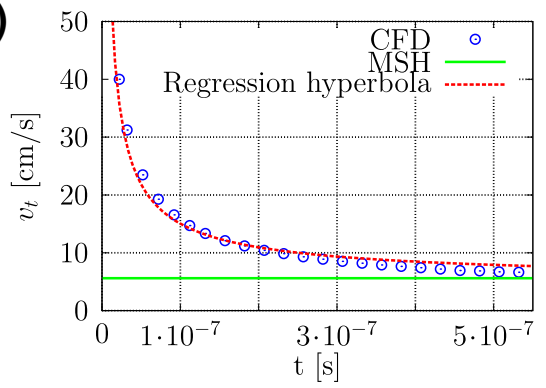

Fig. 6. (a) Computational domain and mesh: $l_{D}=1.75 \mu \mathrm{m}$, the inner rectangle $\left(l_{D} \times 1.25 l_{D}\right)$ has a grid resolution of $200 \times 250$ cells, all boundaries are adiabatic. (b) Numerically calculated tip velocity converges to the theoretical steady-state velocity from the marginal stability hypothesis for an initial supercooling in the liquid phase of $\Delta T=10 \mathrm{~K}$.
Table 1

Steady state tip velocity for $\Delta T=10 \mathrm{~K}$ : mesh convergence study.

\begin{tabular}{llll}
\hline $\begin{array}{l}\text { Norm. grid resol., } \Delta x \\
(-)\end{array}$ & $\begin{array}{l}\text { Norm. exec. time, } t_{e x} \\
(-)\end{array}$ & $\begin{array}{l}\text { Tip velocity, } v_{t} \\
(\mathrm{~cm} / \mathrm{s})\end{array}$ & $\begin{array}{l}\text { Error } \\
(\%)\end{array}$ \\
\hline 1 & 1 & 3.24 & 27.8 \\
$1 / 2$ & 6.4 & 3.97 & 11.6 \\
$1 / 4$ & 50.6 & 4.22 & 6.0 \\
\hline
\end{tabular}

of a needle or dendrite and its tip radius at a given initial supercooling degree is given by the solution of Ivantsov [5], $v_{t} r_{t}=$ const., Fig. 1. Introducing the interfacial energy a condition of stability is invoked. An individual selection of the tip radius, $r_{t}$, which emerges for a given supercooling, can be performed using the theoretical approach of the marginal stability hypothesis. To every selected value of the tip radius should correspond an exact steady-state tip velocity value.

The tip velocities and radii of several paraboloidal needles are analyzed numerically and compared to the theoretical ones. The interfacial energy considered in the following simulations corresponds to $\sigma=0.028 \mathrm{~kg} / \mathrm{s}^{2}$. Both kinetic effects and anisotropies of surface energy are neglected. The numerical steady-state tip velocity is obtained by computing a needle (setting the initial tip radius) growing into a supercooled liquid. All boundaries of the domain are adiabatic. The rectangle into which the needle is growing, Fig. 6(a), has a grid resolution of $200 \times 250$ cells. $l_{D}$ represents the length of the initial needle. The rest of the domain is meshed using a coarser grid. At the beginning, the tip of the needle moves fast due to the high temperature gradient at the interface, since the liquid's temperature at $t=0$ is spatially constant at $T_{l}=T_{m}-\Delta T$. A smooth temperature field develops with time and the high temperature gradient diminishes until the steady-state tip velocity is reached.

The steady state is calculated by adopting the domain depicted in Fig. 6 (a) until, firstly, the temperature at the left, right or top boundary deviates more than $0.1 \%$ from the initial supercooling and, secondly, the deviation of the initial tip curvature is more then $5 \%$. A deviation from the initial supercooling at the boundaries violates the assumption of an infinite half-space into which the needle is growing, whereas a deviation from the initial tip curvature would violate the marginal stability constraint. Fig. 6(b) shows the numerically calculated tip velocities, the regression hyperbola which extrapolates the numerical values and the steady-state velocity from the marginal stability hypothesis for $\Delta T=10 \mathrm{~K}$. Table $1 \mathrm{com}-$ prises the calculated tip velocity at a supercooling of $10 \mathrm{~K}$ for different grid resolutions. The velocity predicted by utilizing the

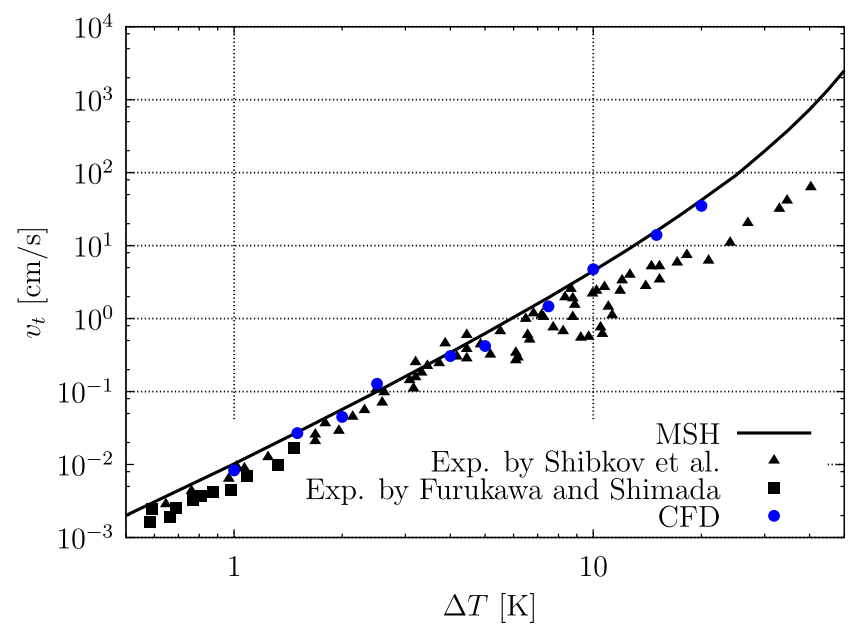

Fig. 7. Comparison of the numerical results with the marginal stability hypothesis. 
marginal stability hypothesis amounts to $4.49 \mathrm{~cm} / \mathrm{s}$. By decreasing the grid resolution, the velocity converges to the theoretical value.

This tip radius predicted theoretically is used to set up a family of computations of paraboloids growing into water under different undercoolings. The steady state tip velocity is then compared with the velocity predicted by the marginal stability hypothesis. Fig. 7 shows the tip velocity in terms of the supercooling obtained from the marginal stability theory and computed using the present numerical algorithm. The numerical results exhibit very close agreement with the marginal stability theory. A comparison with the experimental data of Furukawa and Shimada [12] and Shibkov et al. [15] reveals good agreement with the marginal stability theory within the diffusion-driven growth region. At high supercoolings $(\Delta T \geqslant 10 \mathrm{~K})$, there is an essential departure (attributed to the kinetic effects) of the prediction, performed by using the diffusional model of dendritic growth, from experimental data.

\section{Conclusions}

A new computational model based on the level set approach for numerically solving the model equations describing thermodynamically-driven processes of the solidification phenomenon of the supercooled water is presented. Kinetic effects and flow within the liquid phase have been presently neglected in the formulation. The corresponding heat transfer equations for both the liquid and the solid phase are solved independently of each other. The temperature fields are coupled through two boundary conditions at the interface: the first boundary condition assumes a constant temperature as a result of the Gibbs-Thomson relation, whereas the second boundary condition represents the velocity of the moving interface resulting from the Stefan heat flux balance. A ghostface method is applied to impose a Dirichlet boundary condition at the interface (in relation to the Gibbs-Thomson effect) and to ensure accurate calculation of the temperature normal derivative which is necessary to fulfill the heat flux balance at the interface. Within a narrow band around the interface, whose width is temporally adjusted to the maximum curvature of the interface, the normal-to-interface velocity is appropriately expanded. This velocity is used to update the level set function within the afore-mentioned narrow-band.

The computational model can capture both the stable and the unstable stage of supercooled water solidification. Validation of the computational model yields an excellent agreement between the present computations and the theoretical results of the supercooled Stefan's freezing model for planar solidification (describing the propagation of an unperturbed interface). In the case of slightly perturbed interfaces, the morphological instability of solidification front has been quantitatively checked in comparison to the Mullins-Sekerka theory, resulting in an agreement within a few percent. Concerning the growth of dendrites (unstable freezing), two-dimensional simulations show that the computational model converges to exact solutions of the crystal growth theory of Ivantsov [5]. Including capillary forces (in terms of surface tension), the steady state tip velocity of computed needles converges to the selection theory of Oldfield [16] and Langer and Müller-Krumbhaar [18]. The numerical results exhibit very close agreement with the marginal stability theory. Furthermore, a comparison with the experimental data of Furukawa and Shimada [12] and Shibkov et al. [15] reveals very good agreement within the diffusion-driven growth region.

\section{Acknowledgements}

This research was supported by the German Scientific Foundation (DFG) in the framework of the SFB-TRR 75 collaborative research center.

\section{References}

[1] B.C. Bernstein, C. Le Bot, An inferred climatology of icing conditions aloft including supercooled large drops. part II: Europe, Asia, and the Globe, J. Appl. Meteor. Climatol. 48 (2009) 1503.

[2] S.G. Cober, A.I. George, Characterization of aircraft icing environments with supercooled large drops for application to commercial aircraft certification, J. Appl. Meteor. Climatol. 51 (2012) 265-284.

[3] J. Kepler, Ioannis Kepleri,. strena sev de nive sexangula, Godfrey Tampach, Frankfurt am Main, 1611.

[4] U. Nakaya, Snow Crystals: Natural and Artificial, Harvard Univ. Press, Cambridge, 1954.

[5] G. Ivantsov, Temperature field around spherical, cylindrical, and needleshaped crystals which grow in supercooled melt, Dokl. Akad. Nauk SSSR 558 (1947) 567-569.

[6] A. Karma, Y. Lee, M. Plapp, Three dimensional dendrite tip morphology at low undercooling, Phys. Rev. E 61 (2000) 3996-4006.

[7] J.S. Langer, Dendrites, viscous fingers, and the theory of pattern formation, Science 243 (1989) 1150-1156.

[8] W.W. Mullins, R.F. Sekerka, Morphological stability of a particle growing by diffusion or heat flow, J. Appl. Phys. 34 (1963) 323-329.

[9] W.W. Mullins, R.F. Sekerka, The stability of a planar interface during solidification of a dilute binary alloy, J. Appl. Phys. 35 (1964) 444-451.

[10] H. Sakagushi, M. Ohtaki, A coupled map lattice model for dendritic patterns, Physica A 272 (1999) 300-313.

[11] V. Alexiades, A.D. Solomon, Mathematical Modeling of Melting and Freezing Processes, Hemisphere Publishing Corporation, Washington, 1993.

[12] Y. Furukawa, W. Shimada, Three-dimensional pattern formation during growth of ice dendrites - its relation to universal law of dendritic growth, J. Cryst. Growth 128 (1993) 234-239.

[13] A.A. Shibkov, Y.I. Golovin, M.A. Zheltov, A.A. Korolev, A.A. Vlasov, Kinetics and morphology of nonequilibrium growth of ice in supercooled water, Crystallogr. Rep. 46 (2001) 496-502.

[14] A.A. Shibkov, Y.I. Golovin, M.A. Zheltov, A.A. Korolev, A.A. Leonov, Morphology diagram of nonequilibrium patterns of ice crystals growing in supercooled water, Physica A 319 (2003) 65-79.

[15] A.A. Shibkov, M.A. Zheltov, A.A. Korolev, A.A. Kazakov, A.A. Leonov, Crossover from diffusion-limited to kinetics-limited growth of ice crystals, J. Cryst. Growth 285 (2005) 215-227.

[16] W. Oldfield, Computer model studies of dendritic growth, Mater. Sci. Eng. 11 (1973) 211-218.

[17] J.S. Langer, R.F. Müller-Krumbhaar, Stability effects in dendritic crystal growth, J. Cryst. Growth 42 (1977) 11-14.

[18] J.S. Langer, R.F. Müller-Krumbhaar, Theory of dendritic growth-I. Elements of a stability analysis, Acta Metall. 26 (1978) 1681-1687.

[19] J.S. Langer, R.F. Müller-Krumbhaar, Theory of dendritic growth-II. Instabilities in the limit of vanishing surface tesnion, Acta Metall. 26 (1978) 1689-1695.

[20] J.S. Langer, R.F. Müller-Krumbhaar, Theory of dendritic growth-III. Effects of surface tension, Acta Metall. 26 (1978) 1697-1708.

[21] R. Kobayashi, Modeling and numerical simulations of dendritic crystal growth, Phys. D: Nonlinear Phenomena 63 (1993) 410-423.

[22] A. Karma, W.-J. Rappel, Phase-field simulation of three-dimensional dendrites: is microscopic solvability theory correct?, J Cryst. Growth 174 (1997) 54-64.

[23] Y.-T. Kim, N. Goldenfeld, J. Dantzig, Computation of dendritic microstructures using a level set method, Phys. Rev. E 62 (2000) 2471-2474.

[24] A.A. Wheeler, B.T. Murray, R. Schaefer, Computation of dendrites using a phase field model, Physica D 66 (1993) 243-262.

[25] M. Do-Quang, G. Amberg, Simulation of free dendritic crystal growth in a gravity environment, J. Comput. Phys. 227 (2008) 1772-1789.

[26] S. Osher, J.A. Sethian, Fronts propagating with curvature-dependent speed: Algorithms based on hamilton-jacobi formulations, J. Comput. Phys. 79 (1988) 12-49.

[27] M. Sussman, P. Smereka, S. Osher, A level set approach for computing solutions to incompressible two-phase flow, J. Comput. Phys. 114 (1994) 146-159.

[28] S.A. Sethian, A fast marching level set method for monotonically advancing fronts, Proc. Natl. Acad. Sci. USA 93 (1996) 1591-1595.

[29] J.A. Sethian, Level Set Methods and fast Marching Methods, Cambridge University Press, Cambridge, 1999.

[30] S.A. Sethian, J. Strain, Crystal growth and dendritic solidification, J. Comput. Phys. 98 (1992) 231-253.

[31] S. Chen, B. Merriman, S. Osher, P. Smereka, A simple level-set method for solving stefan problems, J. Comput. Phys. 135 (1997) 8-29.

[32] S.H. Davis, Theory of Solidification, Cambridge University Press, Cambridge, 2001.

[33] S.-C. Huang, M.E. Glicksman, Fundamentals of dendritic solidification: I. Steady-state tip-growth, Acta Metall. Mater. 29 (1981) 701-716.

[34] S.-C. Huang, M.E. Glicksman, Fundamentals of dendritic solidification: Development of sidebranch structure, Acta Metall. Mater. 29 (1981) 717-731.

[35] F. Gibou, R.P. Fedkiw, L.T. Cheng, M. Kang, A second-order-accurate symmetric discretization of the poisson equation on irregular domains, J. Comput. Phys. 176 (2002) 205-227. 